\title{
Harmonic Analysis of Translation Invariant Valuations
}

\author{
Semyon Alesker, Andreas Bernig and Franz E. Schuster
}

\begin{abstract}
The decomposition of the space of continuous and translation invariant valuations into a sum of $\mathrm{SO}(n)$ irreducible subspaces is obtained. A reformulation of this result in terms of a Hadwiger type theorem for continuous translation invariant and $\mathrm{SO}(n)$-equivariant tensor valuations is also given. As an application, symmetry properties of rigid motion invariant and homogeneous bivaluations are established and then used to prove new inequalities of Brunn-Minkowski type for convex body valued valuations.
\end{abstract}

\section{Introduction and statement of main results}

Let $V$ be an $n$-dimensional Euclidean vector space and let $A$ be an abelian semigroup. A function $\phi$ defined on convex bodies (compact convex sets) in $V$ and taking values in $A$ is called a valuation, or additive, if

$$
\phi(K)+\phi(L)=\phi(K \cup L)+\phi(K \cap L)
$$

whenever $K, L$ and $K \cup L$ are convex.

The most important cases are $A=\mathbb{R}$ or $\mathbb{C}$ (scalar valued valuations), $A=\operatorname{Sym}^{k} V$ (tensor valuations) and $A=\mathcal{K}^{n}$, the semigroup of convex bodies in $V$ with the Minkowski addition (Minkowski valuations).

Scalar valued valuations play an important role in integral geometry. Hadwiger characterized in [27] the continuous Euclidean motion invariant valuations. Almost all classical integral-geometric formulas can be reduced to this landmark result. For generalizations of this idea in different directions, we refer to $[3,5,12,16,18,31,37,47$.

Tensor valuations were studied by McMullen [48], the first author [4] and Ludwig [33]. Recently, a full set of kinematic formulas for tensor valuations was obtained by Hug, Schneider and R. Schuster [28, 29].

The best known example of a Minkowski valuation is the projection body. This central notion from affine geometry has many applications in several areas such as geometric tomography, stereology, computational geometry, optimization or functional analysis. For a systematic study of Minkowski valuations, we refer to $[\mathbf{2 3}, \mathbf{2 4}, \mathbf{3 0}, \mathbf{3 2}-\mathbf{3 5}, \mathbf{5 1}]$ and the references therein. 
In this article, we contribute to these three different directions in the theory of valuations. Our main result may be stated in the language of scalar valued valuations or in the language of tensor valuations. For simplicity, we assume throughout this paper that $n \geq 3$, even if most of the results also hold true for $n=2$.

A valuation $\phi$ is called translation invariant if $\phi(K+x)=\phi(K)$ for all $x \in V$ and $K \in \mathcal{K}^{n}$ and $\phi$ is said to have degree $i$ if $\phi(t K)=t^{i} \phi(K)$ for all $K \in \mathcal{K}^{n}$ and $t>0$. We call $\phi$ even if $\phi(-K)=\phi(K)$ and odd if $\phi(-K)=-\phi(K)$ for all $K \in \mathcal{K}^{n}$. We denote by Val the vector space of all continuous translation invariant complex valued valuations and we write $\mathbf{V a l}_{i}^{ \pm}$for its subspace of all valuations of degree $i$ and even/odd parity. An important result by McMullen [46] is that

$$
\mathbf{V a l}=\bigoplus_{0 \leq i \leq n}\left(\mathrm{Val}_{i}^{+} \oplus \mathbf{V a l}_{i}^{-}\right)
$$

In order to state our main theorem, we need the following basic fact from the representation theory of the group $\mathrm{SO}(n)$ : The isomorphism classes of irreducible representations of $\mathrm{SO}(n)$ are parametrized by their highest weights, namely sequences of integers $\left(\lambda_{1}, \lambda_{2}, \ldots, \lambda_{\lfloor n / 2\rfloor}\right)$ such that

$$
\begin{cases}\lambda_{1} \geq \lambda_{2} \geq \ldots \geq \lambda_{\lfloor n / 2\rfloor} \geq 0 & \text { for odd } n \\ \lambda_{1} \geq \lambda_{2} \geq \ldots \geq \lambda_{n / 2-1} \geq\left|\lambda_{n / 2}\right| & \text { for even } n .\end{cases}
$$

(See Section 3 for the background material from representation theory.)

The natural action of the group $\mathrm{SO}(n)$ on the space Val is given by

$$
(\vartheta \phi)(K)=\phi\left(\vartheta^{-1} K\right), \quad \vartheta \in \mathrm{SO}(n), \phi \in \mathbf{V a l} .
$$

Our main theorem is the following decomposition of the space Val into irreducible $\mathrm{SO}(n)$-modules.

Theorem 1. Let $0 \leq i \leq n$. The space $\mathbf{V a l}_{i}$ is the direct sum of the irreducible representations of $\mathrm{SO}(n)$ with highest weights $\left(\lambda_{1}, \ldots, \lambda_{\lfloor n / 2\rfloor}\right)$ precisely satisfying the following additional conditions:

(i) $\lambda_{j}=0$ for $j>\min \{i, n-i\}$;

(ii) $\left|\lambda_{j}\right| \neq 1$ for $1 \leq j \leq\lfloor n / 2\rfloor$;

(iii) $\left|\lambda_{2}\right| \leq 2$.

In particular, under the action of $\mathrm{SO}(n)$ the space $\mathbf{V a l}_{i}$ is multiplicity free. 
Earlier versions of Theorem 1 for even valuations were obtained in [5] and [10. These results were subsequently applied in the construction of new algebraic structures on the space $\mathbf{V a l}$ (see [7, 15]) which provided the means for a fuller understanding of the integral geometry of compact groups acting transitively on the unit sphere (see e.g. [6, 12, 16, 18]).

For the proof of Theorem 11 we draw on methods from representation theory, differential geometry and geometric measure theory. To be more specific, we use a representation of smooth translation invariant valuations via integral currents first obtained in [8] and later refined in [14] and [13] as well as an analysis of the action of $\mathrm{SO}(n)$ on the space of translation invariant differential forms on a contact manifold (see Sections 4 and 5).

We now state a reformulation of Theorem 1 in the language of tensor valuations. Let $(\Gamma, \varrho)$ be a (finite dimensional, complex) representation of $\mathrm{SO}(n)$. A continuous translation invariant valuation with values in $\Gamma$ is called $\mathrm{SO}(n)$ equivariant if

$$
\phi(\vartheta K)=\varrho(\vartheta) \phi(K)
$$

for all $\vartheta \in \mathrm{SO}(n)$ and $K \in \mathcal{K}^{n}$.

Theorem 1'. Let $(\Gamma, \varrho)$ be an irreducible $\mathrm{SO}(n)$ representation and let $0 \leq i \leq n$. There exists a non-trivial continuous translation invariant and $\mathrm{SO}(n)$ equivariant valuation of degree $i$ with values in $\Gamma$ if and only if the highest weight of $\Gamma$ satisfies the conditions (i)-(iii) from Theorem 1 . This valuation is unique up to scaling.

Since a finite dimensional representation of $\mathrm{SO}(n)$ can be decomposed into a sum of irreducible representations, Theorem $1^{\prime}$ can be used to study the space of equivariant $\Gamma$-valued valuations also for reducible $\Gamma$ (compare the examples in Section 5).

The case of symmetric tensors, namely $\Gamma=\operatorname{Sym}^{k} V$, has been intensively treated in [3, 28, 29, 33, 48. In these papers, translation invariance is replaced by the more general isometry covariance. In the recent article [29], Hug, Schneider and R. Schuster explicitly determined the dimension of the space of all continuous isometry covariant tensor valuations of a fixed rank and of a given degree of homogeneity. However, these computations do not seem to give a basis of the subspace of translation invariant tensor valuations. For the general, non-symmetric, case, not much seems to be known except the construction of $\Lambda^{k} V \otimes \Lambda^{k} V$-valued translation invariant valuations in [11]. 
Definition. A map $\varphi: \mathcal{K}^{n} \times \mathcal{K}^{n} \rightarrow \mathbb{C}$ is called a bivaluation if $\varphi$ is additive in each argument. A bivaluation $\varphi$ is called translation biinvariant if $\varphi$ is invariant under independent translations of its arguments and $\varphi$ is said to have bidegree $(i, j)$ if $\varphi(t K, s L)=t^{i} s^{j} \varphi(K, L)$ for all $K, L \in \mathcal{K}^{n}$ and $t, s>0$. We say $\varphi$ is $\mathrm{O}(n)$ invariant (resp. $\mathrm{SO}(n)$ invariant) if $\varphi(\vartheta K, \vartheta L)=\varphi(K, L)$ for all $K, L \in \mathcal{K}^{n}$ and $\vartheta \in \mathrm{O}(n)$ (resp. $\left.\vartheta \in \mathrm{SO}(n)\right)$.

In their book on geometric probability, Klain and Rota [31] pose the problem to classify all "invariant" bivaluations. First such classification results were obtained recently by Ludwig [36]. In Section 6 we obtain a description of all continuous translation biinvariant bivaluations which can be seen as a starting point for systematic investigations of this problem.

As an application of Theorem 1, we obtain the following important symmetry property of rigid motion invariant homogeneous bivaluations.

Theorem 2. If $\varphi: \mathcal{K}^{n} \times \mathcal{K}^{n} \rightarrow \mathbb{R}$ is a continuous translation biinvariant and $\mathrm{O}(n)$ invariant bivaluation of bidegree $(i, i), 0 \leq i \leq n$, then

$$
\varphi(K, L)=\varphi(L, K)
$$

for every $K, L \in \mathcal{K}^{n}$.

As a byproduct of our proof of Theorem 2, we also obtain that if the bivaluation $\varphi$ is as above but merely $\mathrm{SO}(n)$ invariant, then (1.2) still holds true if $(i, n) \neq(2 k+1,4 k+2), k \in \mathbb{N}$. If $n \equiv 2 \bmod 4$, then there exist continuous translation biinvariant and $\mathrm{SO}(n)$ invariant bivaluations of bidegree $\left(\frac{n}{2}, \frac{n}{2}\right)$ which are not symmetric.

The symmetry property established in Theorem 1 in combination with techniques developed by Lutwak [38, 42] can be used to obtain geometric inequalities for Minkowski valuations. Recall that a map $\Phi: \mathcal{K}^{n} \rightarrow \mathcal{K}^{n}$ is called a Minkowski valuation if $\Phi$ is additive with respect to the usual Minkowski addition of convex sets. We denote by $\mathbf{M V a l}{ }_{i}$ the set of all continuous translation invariant Minkowski valuations of degree $i$.

A convex body $K$ is uniquely determined by its support function $h(K, u)=\max \{u \cdot x: x \in K\}$, for $u \in S^{n-1}$. Among the most important examples of Minkowski valuations is the projection operator $\Pi \in \mathbf{M V a l}_{n-1}$ : The projection body $\Pi K$ of $K$ is the convex body defined by

$$
h(\Pi K, u)=\operatorname{vol}_{n-1}\left(K \mid u^{\perp}\right), \quad u \in S^{n-1},
$$

where $K \mid u^{\perp}$ denotes the projection of $K$ onto the hyperplane orthogonal to $u$. For the special role of the map $\Pi$ in the theory of valuations we refer to $\mathbf{3 2}$. 
Let $0 \leq i \leq n-1$. If $\Phi_{i} \in \mathbf{M V a l}_{i}$ is $\mathrm{O}(n)$ equivariant, i.e. $\Phi_{i}(\vartheta K)=\vartheta \Phi_{i} K$ for all $K \in \mathcal{K}^{n}$ and $\vartheta \in \mathrm{O}(n)$, then the map

$$
\varphi(K, L)=V\left(\Phi_{i} K, L[i], B[n-i-1]\right), \quad K, L \in \mathcal{K}^{n},
$$

where $V\left(\Phi_{i} K, L[i], B[n-i-1]\right)$ denotes the mixed volume of $\Phi_{i} K, i$ copies of $L$ and $n-i-1$ copies of the Euclidean unit ball $B$, is a translation biinvariant and $\mathrm{O}(n)$ invariant bivaluation of bidegree $(i, i)$. By Theorem 2 , it is symmetric in $K$ and $L$.

In the particular case where $i=n-1$ and $\Phi_{n-1}=\Pi$, this symmetry property is well known. Its variants and generalizations have been used extensively for establishing geometric inequalities related to convex and star body valued valuations (see $[20,22,25,38,39,41-45,51]$ and Section 7). Complex versions of the projection body were recently studied in [1], they satisfy similar symmetry properties.

In the following we give one example of the type of inequalities that can be derived from Theorem 2. To this end let us recall a version of the classical Brunn-Minkowski inequality. For $i \in\{0, \ldots, n\}$, let $V_{i}(K)$ denote the $i$-th intrinsic volume of $K \in \mathcal{K}^{n}$. The Brunn-Minkowski inequality for intrinsic volumes states the following: If $2 \leq i \leq n$ and $K, L \in \mathcal{K}^{n}$ have non-empty interior, then

$$
V_{i}(K+L)^{1 / i} \geq V_{i}(K)^{1 / i}+V_{i}(L)^{1 / i},
$$

with equality if and only if $K$ and $L$ are homothetic.

In [38, 42] Lutwak obtained inequalities of Brunn-Minkowski type for a well known family of Minkowski valuations derived from the projection body operator. As an application of Theorem 2, we show that inequalities (1.3) and Lutwak's inequalities for derived projection operators of order $i$ are in fact part of a larger family of Brunn-Minkowski type inequalities which hold for all continuous translation invariant and $\mathrm{SO}(n)$ equivariant Minkowski valuations of a given degree.

Theorem 3. Suppose that $\Phi_{i} \in \mathbf{M V a l}_{i}, 1 \leq i \leq n-1$, is $\mathrm{SO}(n)$ equivariant. If $K, L \in \mathcal{K}^{n}$ have non-empty interior, then

$$
V_{i+1}\left(\Phi_{i}(K+L)\right)^{1 / i(i+1)} \geq V_{i+1}\left(\Phi_{i} K\right)^{1 / i(i+1)}+V_{i+1}\left(\Phi_{i} L\right)^{1 / i(i+1)} .
$$

If $i \geq 2$ and $\Phi_{i}$ maps convex bodies with non-empty interior to bodies with non-empty interior, then equality holds if and only if $K$ and $L$ are homothetic.

The special case of Theorem 3 for even Minkowski valuations was recently established by other methods by the third author [51]. 


\section{Translation invariant valuations}

In the following we collect background results on translation invariant complex valued valuations needed in subsequent sections. In particular, we recall the definition of $\mathrm{O}(n)$ finite valuations and smooth valuations as well as their representation via integral currents.

A classical theorem of Minkowski states that the volume of a Minkowski linear combination $t_{1} K_{1}+\ldots+t_{k} K_{k}$ of convex bodies $K_{1}, \ldots, K_{k}$ can be expressed as a homogeneous polynomial of degree $n$ :

$$
V_{n}\left(t_{1} K_{1}+\ldots+t_{k} K_{k}\right)=\sum_{i_{1}, \ldots, i_{n}=1}^{k} V\left(K_{i_{1}}, \ldots, K_{i_{n}}\right) t_{i_{1}} \cdots t_{i_{n}} .
$$

The coefficients $V\left(K_{i_{1}}, \ldots, K_{i_{n}}\right)$ are called mixed volumes of $K_{i_{1}}, \ldots, K_{i_{n}}$. Clearly, $V(K, \ldots, K)=V_{n}(K)$. Moreover, mixed volumes are symmetric, non-negative and multilinear with respect to Minkowski linear combinations. They are also continuous with respect to the Hausdorff metric and satisfy the following two properties:

- If $K, L \in \mathcal{K}^{n}$ such that $K \cup L \in \mathcal{K}^{n}$, and $\mathbf{C}=\left(K_{1}, \ldots, K_{i}\right)$, then

$$
V_{i}(K, \mathbf{C})+V_{i}(L, \mathbf{C})=V_{i}(K \cup L, \mathbf{C})+V_{i}(K \cap L, \mathbf{C})
$$

where $V_{i}(K, \mathbf{C})$ denotes the mixed volume $V\left(K, \ldots, K, K_{1}, \ldots, K_{i}\right)$.

- Mixed volumes are invariant under independent translations of their arguments and they are invariant under simultaneous unimodular linear transformations, i.e., if $K_{1}, \ldots, K_{n} \in \mathcal{K}^{n}$ and $A \in \mathrm{SL}(n)$, then

$$
V\left(A K_{1}, \ldots, A K_{n}\right)=V\left(K_{1}, \ldots, K_{n}\right) .
$$

Recall that we denote by Val the vector space of continuous translation invariant complex valued valuations and we write $\mathbf{V a l}_{i}^{ \pm}$for its subspaces of all valuations of degree $i$ and even/odd parity.

It is easy to see that the space $\mathbf{V a l}_{0}$ is one-dimensional. The analogous (non-trivial) statement for $\mathbf{V a l}_{n}$ was proved by Hadwiger [27, p. 79].

The following consequence of McMullen's decomposition (1.1) is well known. 
Corollary 2.1. Let $C \in \mathcal{K}^{n}$ be a fixed convex body with non-empty interior. The space Val becomes a Banach space under the norm

$$
\|\phi\|=\sup \{|\phi(K)|: K \subseteq C\} .
$$

Moreover, a different choice of $C$ yields an equivalent norm.

The natural continuous action of the group $\mathrm{GL}(n)$ on the Banach space Val is given by

$$
(A \phi)(K)=\phi\left(A^{-1} K\right), \quad A \in \mathrm{GL}(n), \phi \in \text { Val. }
$$

Note that the subspaces $\mathbf{V a l}_{i}^{ \pm} \subseteq \mathbf{V a l}$ are invariant under this $\mathrm{GL}(n)$ action.

The following result is known as the Irreducibility Theorem. It implies a conjecture by McMullen that the linear combinations of mixed volumes form a dense subspace in Val.

Theorem 2.2. ([5]) The natural action of $\mathrm{GL}(n)$ on $\mathbf{V a l}_{i}^{ \pm}$is irreducible for every $i \in\{0, \ldots, n\}$.

In the following it will be important for us to work with two different dense subsets of valuations in Val:

Definition $A$ valuation $\phi \in$ Val is called $\mathrm{O}(n)$ finite if the $\mathrm{O}(n)$ orbit of $\phi$, i.e. the subspace $\operatorname{span}\{\vartheta \phi: \vartheta \in \mathrm{O}(n)\}$, is finite dimensional.

A valuation $\phi \in \mathbf{V a l}$ is called smooth if the map $\mathrm{GL}(n) \rightarrow$ Val defined by $A \mapsto A \phi$ is infinitely differentiable.

The notions of $\mathrm{O}(n)$ finite and smooth valuations are special cases of more general well known concepts from representation theory (see e.g. [52]).

We denote the space of continuous translation invariant and $\mathrm{O}(n)$ finite valuations by $\mathbf{V a l}^{f}$ and we write $\mathbf{V a l}^{\infty}$ for the space of smooth translation invariant valuations. For the subspaces of homogeneous valuations of given parity in $\mathbf{V a l}^{f}$ and $\mathbf{V a l}{ }^{\infty}$ we write $\mathbf{V a l}_{i}^{ \pm, f}$ and $\mathbf{V a l}{ }_{i}^{ \pm, \infty}$, respectively.

It is well known (cf. [17, p. 141] and [52, p. 32]) that the set of $\mathrm{O}(n)$ finite valuations $\mathbf{V a l}_{i}^{ \pm, f}$ is a dense $\mathrm{O}(n)$ invariant subspace of $\mathbf{V a l}_{i}^{ \pm}$and that the set of smooth valuations $\mathbf{V a l}_{i}^{ \pm, \infty}$ is a dense $\mathrm{GL}(n)$ invariant subspace of $\mathbf{V a l}_{i}^{ \pm}$. Moreover, $\mathbf{V a l}^{f} \subseteq \mathbf{V a l}^{\infty}$ and from (1.1) one easily deduces that the spaces $\mathbf{V a l}^{f}$ and $\mathbf{V a l}^{\infty}$ admit direct sum decompositions into their corresponding subspaces of homogeneous valuations of given parity. 
An equivalent description of smooth valuations can be given in terms of the normal cycle map. Let $S V=V \times S^{n-1}$ denote the unit sphere bundle on $V$. For $K \in \mathcal{K}^{n}$ and $x \in \partial K$, we write $N(K, x)$ for the normal cone of $K$ at $x$. The normal cycle (or generalized normal bundle) of a convex body $K$ is the Lipschitz submanifold of $S V$ defined by

$$
\operatorname{nc}(K)=\{(x, u) \in S V: x \in \partial K, u \in N(K, x)\} \text {. }
$$

For $0 \leq i \leq n-1$, let $\Omega^{i, n-i-1}$ denote the space of smooth translation invariant differential forms of bidegree $(i, n-i-1)$ on $S V$. The following result is a special case of [8, Theorem 5.2.1]:

Lemma 2.3. If $0 \leq i \leq n-1$, then the map $\nu: \Omega^{i, n-i-1} \rightarrow \mathbf{V a l}_{i}^{\infty}$, defined by

$$
\nu(\omega)(K)=\int_{\operatorname{nc}(K)} \omega
$$

is surjective.

The kernel of the map $\nu$ was described in [14] in terms of the Rumin operator [49], a second order differential operator which acts on smooth forms on the sphere bundle. A refined version of this result (stated in Section 4 as Theorem 4.3) was recently proved in [13] and will be crucial in the proof of Theorem 1. We also remark that recently a broader notion of smooth valuations in the setting of smooth manifolds was introduced, see [8]. The classical concept of valuations as used in this article is in some sense an infinitesimal version of this more general notion.

The description of smooth valuations provided by Lemma 2.3 was the main tool used in [14 to establish a Hard Lefschetz Theorem for translation invariant valuations (see also [6, 9]). The next statement is an immediate consequence of this result:

Theorem 2.4. For every $i \in\{0, \ldots, n\}$, the spaces $\mathbf{V a l}_{i}^{\infty}$ and $\mathbf{V a l}_{n-i}^{\infty}$ are isomorphic as $\mathrm{SO}(n)$ modules.

\section{Irreducible representations of $\mathrm{SO}(n)$ and $\mathrm{O}(n)$}

In this section we recall some well known results concerning irreducible representations of the groups $\mathrm{SO}(n)$ and $\mathrm{O}(n), n \geq 3$. As a general reference for this material we recommend the books by Bröcker and tom Dieck [17], Fulton and Harris [19], and Goodman and Wallach [21]. 
Since $\mathrm{SO}(n)$ and $\mathrm{O}(n)$ are compact Lie groups, all their irreducible representations are finite dimensional. The equivalence classes of irreducible complex representations of $\mathrm{SO}(n)$ are indexed by their highest weights, namely $\lfloor n / 2\rfloor$-tuples of integers $\left(\lambda_{1}, \lambda_{2}, \ldots, \lambda_{\lfloor n / 2\rfloor}\right)$ such that

$$
\begin{cases}\lambda_{1} \geq \lambda_{2} \geq \ldots \geq \lambda_{\lfloor n / 2\rfloor} \geq 0 & \text { for odd } n \\ \lambda_{1} \geq \lambda_{2} \geq \ldots \geq \lambda_{n / 2-1} \geq\left|\lambda_{n / 2}\right| & \text { for even } n .\end{cases}
$$

We refer to [17] or 2] for an introduction to highest weights.

In the following we use $\Gamma_{\lambda}$ to denote any isomorphic copy of an irreducible representation of $\mathrm{SO}(n)$ with highest weight $\lambda=\left(\lambda_{1}, \lambda_{2}, \ldots, \lambda_{\lfloor n / 2\rfloor}\right)$.

\section{Examples:}

(a) The only one dimensional (complex) representation of $\mathrm{SO}(n)$ is the trivial representation; it corresponds to the $\mathrm{SO}(n)$ module $\Gamma_{(0, \ldots, 0)}$.

(b) We denote by $V_{\mathbb{C}}=V \otimes \mathbb{C}$ the complexification of $V$. The standard representation of $\mathrm{SO}(n)$ on $V_{\mathbb{C}}$ corresponds to $\Gamma_{(1,0, \ldots, 0)}$.

(c) For every $0 \leq i \leq\lfloor n / 2\rfloor-1$, the exterior power $\Lambda^{i} V_{\mathbb{C}}$ is an irreducible $\mathrm{SO}(n)$ module with $\lambda=(1, \ldots, 1,0, \ldots, 0)$, where 1 appears $i$ times.

If $n=2 k+1$ is odd, the exterior power $\Lambda^{k} V_{\mathbb{C}}$ is also irreducible; but if $n=2 k$ is even, it splits as $\Lambda^{k} V_{\mathbb{C}}=\Gamma_{(1, \ldots, 1)} \oplus \Gamma_{(1, \ldots, 1,-1)}$

For every $i \in\{0, \ldots, n\}$, there is a natural isomorphism of $\mathrm{SO}(n)$ modules

$$
\Lambda^{i} V_{\mathbb{C}} \cong \Lambda^{n-i} V_{\mathbb{C}}
$$

(d) For $k \geq 2$, the symmetric power $\mathrm{Sym}^{k} V_{\mathbb{C}}$ is not irreducible as $\mathrm{SO}(n)$ module; its decomposition into irreducible submodules is given by

$$
\operatorname{Sym}^{k} V_{\mathbb{C}}=\bigoplus_{j=0}^{\lfloor k / 2\rfloor} \Gamma_{(k-2 j, 0, \ldots, 0)} .
$$

A description of the irreducible representations of the full orthogonal group $\mathrm{O}(n)$ can be given in terms of the irreducible representations of its identity component $\mathrm{SO}(n)$ (cf. [21, p. 249]). The main difference arises from the fact that $\mathrm{O}(n)$ has a non-trivial one dimensional representation, called the determinant representation, which corresponds to the $\mathrm{O}(n)$ module $\Lambda^{n} V_{\mathbb{C}}$. 
Lemma 3.1. Let $\lambda=\left(\lambda_{1}, \ldots, \lambda_{\lfloor n / 2\rfloor}\right)$ be a tuple of integers satisfying (3.1).

(a) If $n$ is odd, then the irreducible representation $\Gamma_{\lambda}$ of $\operatorname{SO}(n)$ is the restriction of two non-isomorphic irreducible $\mathrm{O}(n)$ representations $\bar{\Gamma}_{\lambda}$ and $\bar{\Gamma}_{\lambda} \otimes \Lambda^{n} V_{\mathbb{C}}$.

(b) If $n$ is even and $\lambda_{n / 2}=0$, then the irreducible representation $\Gamma_{\lambda}$ of $\mathrm{SO}(n)$ is the restriction of two non-isomorphic irreducible $\mathrm{O}(n)$ representations $\bar{\Gamma}_{\lambda}$ and $\bar{\Gamma}_{\lambda} \otimes \Lambda^{n} V_{\mathbb{C}}$. If $\lambda_{n / 2} \neq 0$, then $\Gamma_{\lambda}$ is not such a restriction.

(c) If $n$ is even and $\lambda_{n / 2} \neq 0$, then the $\mathrm{SO}(n)$ representation $\Gamma_{\lambda} \oplus \Gamma_{\lambda^{\prime}}$, where $\lambda^{\prime}:=\left(\lambda_{1}, \ldots, \lambda_{n / 2-1},-\lambda_{n / 2}\right)$, is the restriction of an irreducible $\mathrm{O}(n)$ representation $\bar{\Gamma}_{\lambda}$.

Moreover, all irreducible representations of $\mathrm{O}(n)$ are determined in this way.

Let $\Gamma$ be a (not necessarily irreducible) complex $\mathrm{SO}(n)$ or $\mathrm{O}(n)$ module. Recall that the dual representation is defined on the dual space $\Gamma^{*}$ by

$$
\left(\vartheta u^{*}\right)(v)=u^{*}\left(\vartheta^{-1} v\right), \quad \vartheta \in \mathrm{SO}(n), u^{*} \in \Gamma^{*}, v \in \Gamma .
$$

We say that $\Gamma$ is self-dual if $\Gamma$ and $\Gamma^{*}$ are isomorphic representations. The module $\Gamma$ is called real if there exists a non-degenerate symmetric $\mathrm{SO}(n)$ invariant, or $\mathrm{O}(n)$ respectively, bilinear form on $\Gamma$. In particular, if $\Gamma$ is real, then $\Gamma$ is also self-dual.

The following lemma (cf. [17, p. 292]) will be critical in the proof of Theorem 2 ;

Lemma 3.2. Let $\lambda=\left(\lambda_{1}, \ldots, \lambda_{\lfloor n / 2\rfloor}\right)$ be a tuple of integers satisfying (3.1).

(a) If $n \not \equiv 2 \bmod 4$, then all representations of $\mathrm{SO}(n)$ are real.

(b) If $n \equiv 2 \bmod 4$, then the irreducible representation $\Gamma_{\lambda}$ of $\mathrm{SO}(n)$ is real if and only if $\lambda_{n / 2}=0$. If $\lambda_{n / 2} \neq 0$, then the dual of $\Gamma_{\lambda}$ is $\Gamma_{\lambda^{\prime}}$.

Moreover, all representations of $\mathrm{O}(n)$ are real.

An essential tool in the classification of irreducible modules of a compact group is the character of a representation: Let $\Gamma$ be a finite dimensional (complex) $\mathrm{SO}(n)$ module and let $\varrho: \mathrm{SO}(n) \rightarrow \mathrm{GL}(\Gamma)$ be the corresponding 
representation. The character of $\Gamma$ is the function char $\Gamma: \mathrm{SO}(n) \rightarrow \mathbb{C}$ defined by

$$
(\operatorname{char} \Gamma)(\vartheta)=\operatorname{Tr} \varrho(\vartheta),
$$

where $\operatorname{Tr} \varrho(\vartheta)$ is the trace of the linear map $\varrho(\vartheta): \Gamma \rightarrow \Gamma$.

A complex representation is determined up to isomorphism by its character. Moreover, from properties of the trace map, one immediately obtains several useful arithmetic properties of characters: If $\Gamma$ and $\Theta$ are finite dimensional $\mathrm{SO}(n)$ modules, then

$$
\operatorname{char}(\Gamma \oplus \Theta)=\operatorname{char} \Gamma+\operatorname{char} \Theta
$$

and

$$
\operatorname{char}(\Gamma \otimes \Theta)=\operatorname{char} \Gamma \cdot \operatorname{char} \Theta .
$$

The character of the irreducible $\mathrm{SO}(n)$ modules $\Gamma_{\lambda}$ with highest weights $\lambda=\left(\lambda_{1}, \ldots, \lambda_{\lfloor n / 2\rfloor}\right)$ are described by Weyl's character formula. However, more important for us is a consequence of this description, known as the second determinantal formula, which we describe in the following.

Let $\lambda=\left(\lambda_{1}, \ldots, \lambda_{\lfloor n / 2\rfloor}\right)$ be a tuple of non-negative integers satisfying (3.1). We define the $\mathrm{SO}(n)$ module $\bar{\Gamma}_{\lambda}$ by

$$
\bar{\Gamma}_{\lambda}:= \begin{cases}\Gamma_{\lambda} \oplus \Gamma_{\lambda^{\prime}} & \text { if } n \text { is even and } \lambda_{n / 2} \neq 0 \\ \Gamma_{\lambda} & \text { otherwise. }\end{cases}
$$

The second determinantal formula expresses char $\bar{\Gamma}_{\lambda}$ as a polynomial in the characters $E_{i}$ of the fundamental representations $\Lambda^{i} V_{\mathbb{C}}, i \in \mathbb{Z}$. (Note that $E_{0}=E_{n}=1$ and that we use the convention $E_{i}=0$ for $i<0$ or $i>n$.)

Given a tuple of non-negative integers $\lambda=\left(\lambda_{1}, \ldots, \lambda_{\lfloor n / 2\rfloor}\right)$ satisfying (3.1), recall that the conjugate of $\lambda$ is the $s:=\lambda_{1}$ tuple $\mu=\left(\mu_{1}, \ldots, \mu_{s}\right)$ defined by saying that $\mu_{j}$ is the number of terms in $\lambda$ that are greater than or equal $j$. The second determinantal formula (cf. [19, p. 409]) can be stated as follows:

Theorem 3.3. Let $\lambda=\left(\lambda_{1}, \ldots, \lambda_{\lfloor n / 2\rfloor}\right)$ be a tuple of non-negative integers satisfying (3.1) and let $\mu=\left(\mu_{1}, \ldots, \mu_{s}\right)$ be the conjugate of $\lambda$. The character of $\bar{\Gamma}_{\lambda}$ equals the determinant of the $s \times s$-matrix whose $i$-th row is given by

$$
\left(\begin{array}{llll}
E_{\mu_{i}-i+1} & E_{\mu_{i}-i+2}+E_{\mu_{i}-i} & \cdots & E_{\mu_{i}-i+s}+E_{\mu_{i}-i-s+2}
\end{array}\right) .
$$


It is sometimes convenient for us to take $s>\lambda_{1}$ in the definition of the conjugate of $\lambda$. This just introduces additional zeros at the end of the conjugate tuple. However, note that this does not change the determinant of the matrix defined by (3.6).

In the following we use $\#(\lambda, j)$ to denote the number of terms in a tuple of (non-negative) integers $\lambda=\left(\lambda_{1}, \ldots, \lambda_{\lfloor n / 2\rfloor}\right)$ which are equal to $j$. As a consequence of Theorem [3.3, we note the following auxiliary result which will be needed in the proof of Theorem 1 .

Corollary 3.4. If $i, j \in \mathbb{N}$ are such that $n / 2 \leq i \leq n$ and $i+j \leq n$, then

$$
E_{i} E_{j}-E_{i-1} E_{j-1}=\sum_{\lambda} \operatorname{char} \bar{\Gamma}_{\lambda}
$$

where the sum ranges over all $\lfloor n / 2\rfloor$-tuples of non-negative integers $\lambda=\left(\lambda_{1}, \ldots, \lambda_{\lfloor n / 2\rfloor}\right)$ satisfying (3.1) and

$$
\lambda_{1} \leq 2, \quad \#(\lambda, 1)=n-i-j, \quad \#(\lambda, 2) \leq j .
$$

Proof: If $\lambda=\left(\lambda_{1}, \ldots, \lambda_{\lfloor n / 2\rfloor}\right)$ is a tuple of non-negative integers satisfying (3.1) and (3.8), then the conjugate of $\lambda$ is given by $\mu=\left(\mu_{1}, \mu_{2}\right)$, where $\mu_{2}=\#(\lambda, 2) \leq j$ and $\mu_{1}-\mu_{2}=\#(\lambda, 1)=n-i-j$. Thus, by Theorem 3.3 , the character of $\bar{\Gamma}_{\lambda}$ is given by

$$
\operatorname{char} \bar{\Gamma}_{\lambda}=\operatorname{det}\left(\begin{array}{cc}
E_{\mu_{2}+k} & E_{\mu_{2}+k+1}+E_{\mu_{2}+k-1} \\
E_{\mu_{2}-1} & E_{\mu_{2}}+E_{\mu_{2}-2}
\end{array}\right)
$$

where $k=n-i-j$. Consequently, the right hand side of (3.7) is

$$
\begin{aligned}
\sum_{\lambda} \operatorname{char} \bar{\Gamma}_{\lambda} & =\sum_{\mu_{2}=0}^{j}\left(E_{\mu_{2}+k}\left(E_{\mu_{2}}+E_{\mu_{2}-2}\right)-E_{\mu_{2}-1}\left(E_{\mu_{2}+k+1}+E_{\mu_{2}+k-1}\right)\right) \\
& =E_{n-i} E_{j}-E_{n-(i-1)} E_{j-1} .
\end{aligned}
$$

To finish the proof, note that $E_{n-i}=E_{i}$ by (3.2).

An important class of (infinite dimensional) representations of a Lie group $G$ are those induced from closed subgroups $H$ of $G$. Although in this article we will only need the case $G=\mathrm{SO}(n)$ and $H=\mathrm{SO}(n-1)$, we shall explain this construction for a general compact Lie group $G$ and its closed subgroup $H$. To this end, for any finite dimensional complex vector space $\Gamma$, we denote by $C^{\infty}(G ; \Gamma)$ the space of all smooth functions $f: G \rightarrow \Gamma$. 
If $\Theta$ is any representation of $G$, clearly we obtain a representation $\operatorname{Res}_{H}^{G} \Theta$ of $H$ by restriction. Conversely, each $H$ module $\Gamma$ induces a representation of $G$ as follows: Let $\operatorname{Ind}_{H}^{G} \Gamma \subseteq C^{\infty}(G ; \Gamma)$ be the space of functions defined by

$$
\operatorname{Ind}_{H}^{G} \Gamma:=\left\{f \in C^{\infty}(G ; \Gamma): f(g h)=h^{-1} f(g) \text { for all } g \in G, h \in H\right\} .
$$

The (smooth) induced representation of $G$ on $\operatorname{Ind}_{H}^{G} \Gamma$ is now given by left translation

$$
(g f)(u)=f\left(g^{-1} u\right), \quad g, u \in G .
$$

A basic result on induced representations is the well known Frobenius Reciprocity Theorem (cf. [21, p. 523]):

Theorem 3.5. If $\Theta$ is a $G$ module and $\Gamma$ is an $H$ module, then there is a canonical vector space isomorphism

$$
\operatorname{Hom}_{G}\left(\Theta, \operatorname{Ind}_{H}^{G} \Gamma\right) \cong \operatorname{Hom}_{H}\left(\operatorname{Res}_{H}^{G} \Theta, \Gamma\right) .
$$

Here, $\operatorname{Hom}_{G}$ denotes the space of continuous linear $G$ equivariant maps.

Recall that if $\Theta$ is an irreducible $G$ module, by Schur's lemma, the multiplicity $m(\Xi, \Theta)$ of $\Theta$ in an arbitrary $G$ module $\Xi$ is given by

$$
m(\Xi, \Theta)=\operatorname{dim} \operatorname{Hom}_{G}(\Xi, \Theta)=\operatorname{dim} \operatorname{Hom}_{G}(\Theta, \Xi) .
$$

Thus, by the Frobenius Reciprocity Theorem, if $\Theta$ and $\Gamma$ are irreducible, then the multiplicity of $\Theta$ in $\operatorname{Ind}_{H}^{G} \Gamma$ equals the multiplicity of $\Gamma$ in $\operatorname{Res}_{H}^{G} \Theta$.

In order to apply Theorem 3.5 in our situation, where $G=\operatorname{SO}(n)$ and $H=\mathrm{SO}(n-1)$, we will need a formula for decomposing $\operatorname{Res}_{\mathrm{SO}(n-1)}^{\mathrm{SO}(n)} \Gamma$ into irreducible $\mathrm{SO}(n-1)$ modules. This is the content of the following branching theorem (cf. [19, p. 426]):

Theorem 3.6. If $\Gamma_{\lambda}$, with $\lambda=\left(\lambda_{1}, \ldots, \lambda_{\lfloor n / 2\rfloor}\right)$ satisfying (3.1), is an irreducible representation of $\mathrm{SO}(n)$, then

$$
\operatorname{Res}_{\mathrm{SO}(n-1)}^{\mathrm{SO}(n)} \Gamma_{\lambda}=\bigoplus_{\mu} \Gamma_{\mu}
$$

where the sum ranges over all $\mu=\left(\mu_{1}, \ldots, \mu_{k}\right)$ with $k:=\lfloor(n-1) / 2\rfloor$ and

$$
\begin{cases}\lambda_{1} \geq \mu_{1} \geq \lambda_{2} \geq \mu_{2} \geq \ldots \geq \mu_{k-1} \geq \lambda_{\lfloor n / 2\rfloor} \geq\left|\mu_{k}\right| & \text { for odd } n, \\ \lambda_{1} \geq \mu_{1} \geq \lambda_{2} \geq \mu_{2} \geq \ldots \geq \mu_{k} \geq\left|\lambda_{n / 2}\right| & \text { for even } n .\end{cases}
$$




\section{The Rumin-de Rham complex}

We state in this section a refinement of the description of translation invariant smooth valuations via integral currents. We also establish an auxiliary result which will enable us to subsequently employ the machinery from representation theory explained in Section 3.

Recall that $S V=V \times S^{n-1}$ denotes the unit sphere bundle. The natural smooth (left) action of $\mathrm{SO}(n)$ on $S V$ is given by

$$
l_{\vartheta}(x, u):=(\vartheta x, \vartheta u), \quad \vartheta \in \mathrm{SO}(n),(x, u) \in S V .
$$

Similarly, each $y \in V$ determines a smooth map $t_{y}: S V \rightarrow S V$ by

$$
t_{y}(x, u)=(x+y, u), \quad(x, u) \in S V .
$$

The canonical contact form $\alpha$ on $S V$ is the one form defined by

$$
\left.\alpha\right|_{(x, u)}(w)=\left\langle u, d_{(x, u)} \pi(w)\right\rangle, \quad w \in T_{(x, u)} S V,
$$

where $\pi: S V \rightarrow V$ denotes the canonical projection and $d_{(x, u)} \pi$ its differential at $(x, u) \in S V$. In this way, $S V$ becomes a $2 n-1$ dimensional contact manifold. The kernel of $\alpha$ defines the contact distribution $Q:=\operatorname{ker} \alpha$. The restriction of $d \alpha$ to $Q$ is a non-degenerate two form. In this way, each $Q_{(x, u)}$ becomes a symplectic vector space.

The Reeb vector field $R$ on $S V$ is defined by $R_{(x, u)}=(u, 0)$. It is the unique vector field on $S V$ such that $\alpha(R)=1$ and $i_{R} d \alpha=0$, where $i_{R} d \alpha$ denotes the interior product of $R$ and $d \alpha$. At each point $(x, u), Q_{(x, u)}$ is the orthogonal sum of two copies of $T_{u} S^{n-1}$ and, consequently, we have an orthogonal splitting of the tangent space $T_{(x, u)} S V$ given by

$$
T_{(x, u)} S V=\operatorname{span}_{\mathbb{R}} R_{(x, u)} \oplus T_{u} S^{n-1} \oplus T_{u} S^{n-1} .
$$

The product structure of $S V$ induces a bigrading on the vector space $\Omega^{*}(S V)$ of complex valued smooth differential forms given by

$$
\Omega^{*}(S V)=\bigoplus_{i, j} \Omega^{i, j}(S V)
$$

where $\Omega^{i, j}(S V)$ denotes the subspace of $\Omega^{*}(S V)$ of forms of bidegree $(i, j)$. We write $\Omega^{i, j} \subseteq \Omega^{i, j}(S V)$ for the subspace of translation invariant forms, i.e.,

$$
\Omega^{i, j}=\left\{\omega \in \Omega^{i, j}(S V): t_{y}^{*} \omega=\omega \text { for all } y \in V\right\} .
$$


Here, $t_{y}^{*}$ is the pullback of the map $t_{y}: S V \rightarrow S V$ defined in (4.2). Note that the restriction of the exterior derivative $d$ to $\Omega^{i, j}$ has bidegree $(0,1)$.

The vector space $\Omega^{i, j}$ becomes an $\mathrm{SO}(n)$ module under the (continuous) action

$$
\vartheta \omega=l_{\vartheta^{-1}}^{*} \omega, \quad \vartheta \in \mathrm{SO}(n), \omega \in \Omega^{i, j} .
$$

An important $\mathrm{SO}(n)$ submodule of $\Omega^{i, j}$ is given by the space $\Omega_{v}^{i, j}$ of vertical forms, defined by

$$
\Omega_{v}^{i, j}:=\left\{\omega \in \Omega^{i, j}: \alpha \wedge \omega=0\right\} .
$$

Note that a differential form $\omega \in \Omega^{i, j}$ is vertical if and only if it vanishes on the contact distribution $Q$ of $S V$.

The $\mathrm{SO}(n)$ submodule $\Omega_{h}^{i, j} \subseteq \Omega^{i, j}$ of horizontal forms, is given by

$$
\Omega_{h}^{i, j}:=\left\{\omega \in \Omega^{i, j}: i_{R} \omega=0\right\} \cong \Omega^{i, j} / \Omega_{v}^{i, j} .
$$

It follows from (4.3) and the definition of $\Omega_{h}^{i, j}$ that $\omega \in \Omega^{i, j}$ is horizontal if and only if

$$
\left.\omega\right|_{(x, u)} \in \Lambda^{i} T_{u}^{*} S^{n-1} \otimes \Lambda^{j} T_{u}^{*} S^{n-1} \otimes \mathbb{C}
$$

for every $x \in V$ and each $u \in S^{n-1}$. In the following we will therefore simply write $\left.\omega\right|_{u}$ instead of $\left.\omega\right|_{(x, u)}$ whenever $\omega \in \Omega_{h}^{i, j}$ and $(x, u) \in S V$.

We now fix a point $u_{0} \in S^{n-1}$ and let $\mathrm{SO}(n-1)$ be the stabilizer of $\mathrm{SO}(n)$ at $u_{0}$. For $u \in S^{n-1}$, we denote by $W_{u}:=T_{u} S^{n-1} \otimes \mathbb{C}$ the complexification of the tangent space $T_{u} S^{n-1}$ and we write $W_{0}$ to denote $W_{u_{0}}$.

Lemma 4.1. For $i, j \in \mathbb{N}$, there is an isomorphism of $\mathrm{SO}(n)$ modules

$$
\Omega_{h}^{i, j} \cong \operatorname{Ind}_{\mathrm{SO}(n-1)}^{\mathrm{SO}(n)}\left(\Lambda^{i} W_{0}^{*} \otimes \Lambda^{j} W_{0}^{*}\right) .
$$

Proof: First note that, for each $\vartheta \in \mathrm{SO}(n)$, the differential of the map $l_{\vartheta}: S V \rightarrow S V$ defined in (4.1) induces a linear isomorphism

$$
\widehat{d_{u_{0}} l_{\vartheta}}:=\left(d_{u_{0}} l_{\vartheta}\right)^{*}: \Lambda^{i} W_{\vartheta u_{0}}^{*} \otimes \Lambda^{j} W_{\vartheta u_{0}}^{*} \rightarrow \Lambda^{i} W_{0}^{*} \otimes \Lambda^{j} W_{0}^{*}
$$

Moreover, the natural representation of the group $\mathrm{SO}(n-1)$ on the space $\Lambda^{i} W_{0}^{*} \otimes \Lambda^{j} W_{0}^{*}$ is given by $\eta \mapsto \widehat{d_{u_{0} \eta_{\eta^{-1}}}}$.

Suppose now that $\omega \in \Omega_{h}^{i, j}$. We define $f_{\omega}: \mathrm{SO}(n) \rightarrow \Lambda^{i} W_{0}^{*} \otimes \Lambda^{j} W_{0}^{*}$ by

$$
f_{\omega}(\vartheta)=\widehat{d_{u_{0}} l_{\vartheta}}\left(\left.\omega\right|_{\vartheta u_{0}}\right) .
$$


Clearly, we have $f_{\omega}(\vartheta \eta)=\eta^{-1} f_{\omega}(\vartheta)$ for every $\vartheta \in \operatorname{SO}(n)$ and $\eta \in \operatorname{SO}(n-1)$. This shows that $f_{\omega} \in \operatorname{Ind}_{\mathrm{SO}(n-1)}^{\mathrm{SO}(n)}\left(\Lambda^{i} W_{0}^{*} \otimes \Lambda^{j} W_{0}^{*}\right)$.

Conversely, let $f \in \operatorname{Ind}_{\mathrm{SO}(n-1)}^{\mathrm{SO}(n)}\left(\Lambda^{i} W_{0}^{*} \otimes \Lambda^{j} W_{0}^{*}\right)$. We define a horizontal form $\omega_{f} \in \Omega_{h}^{i, j}$ by

$$
\left.\omega_{f}\right|_{\vartheta u_{0}}={\widehat{d_{u_{0}} l_{\vartheta}}}^{-1}(f(\vartheta)) .
$$

It is not difficult to show that $\omega$ is well defined, i.e. if $\vartheta u_{0}=\vartheta^{\prime} u_{0}$ for some $\vartheta, \vartheta^{\prime} \in \operatorname{SO}(n)$, then $\left.\omega\right|_{\vartheta u_{0}}=\left.\omega\right|_{\vartheta^{\prime} u_{0}}$.

The observation that the $\mathrm{SO}(n)$ equivariant linear maps $\omega \mapsto f_{\omega}$ and $f \mapsto \omega_{f}$ are inverse to each other finishes the proof.

Let $\mathcal{I}^{i, j}$ denote the $\mathrm{SO}(n)$ invariant subspace of $\Omega^{i, j}$ defined by

$$
\mathcal{I}^{i, j}:=\left\{\omega \in \Omega^{i, j}: \omega=\alpha \wedge \xi+d \alpha \wedge \psi, \xi \in \Omega^{i-1, j}, \psi \in \Omega^{i-1, j-1}\right\} .
$$

Finally, we denote by $\Omega_{p}^{i, j}$ the $\mathrm{SO}(n)$ module of primitive forms defined as the quotient

$$
\Omega_{p}^{i, j}:=\Omega^{i, j} / \mathcal{I}^{i, j}
$$

An equivalent description of primitive forms can be given as follows: The multiplication by the symplectic form $-d \alpha$ induces an $\mathrm{SO}(n)$ equivariant linear operator $L: \Omega_{h}^{i, j} \rightarrow \Omega_{h}^{i+1, j+1}$ which is injective if $i+j \leq n-2$. Moreover, it follows from the definition of $\Omega_{p}^{i, j}$ that in this case

$$
\Omega_{p}^{i, j}=\Omega_{h}^{i, j} / L \Omega_{h}^{i-1, j-1} .
$$

From Lemma 4.1 and (4.5), we now immediately obtain

Corollary 4.2. If $i, j \in \mathbb{N}$ are such that $i+j \leq n-1$, then there is an isomorphism of $\mathrm{SO}(n)$ modules

$$
\Omega_{p}^{i, j} \oplus \operatorname{Ind}_{\mathrm{SO}(n-1)}^{\mathrm{SO}(n)}\left(\Lambda^{i-1} W_{0}^{*} \otimes \Lambda^{j-1} W_{0}^{*}\right) \cong \operatorname{Ind}_{\mathrm{SO}(n-1)}^{\mathrm{SO}(n)}\left(\Lambda^{i} W_{0}^{*} \otimes \Lambda^{j} W_{0}^{*}\right) .
$$

Primitive forms are of particular importance for us since the space $\mathbf{V a l}_{i}^{\infty}$ fits into an exact sequence of the spaces $\Omega_{p}^{i, j}$, as was recently established in [13. In order to describe this sequence, note that $d \mathcal{I}^{i, j} \subseteq \mathcal{I}^{i, j+1}$. Thus, by definition (4.4), the exterior derivative, on one hand, induces a linear operator $d_{Q}: \Omega_{p}^{i, j} \rightarrow \Omega_{p}^{i, j+1}$ and, on the other hand, integration over the normal cycle induces a linear map $\nu: \Omega_{p}^{i, n-i-1} \rightarrow \mathbf{V a l}_{i}^{\infty}$ (cf. Lemma 2.3). Clearly, both operators are $\mathrm{SO}(n)$ equivariant. 
Theorem 4.3. If $0 \leq i \leq n$, then there is an exact $\mathrm{SO}(n)$ equivariant sequence of $\mathrm{SO}(n)$ modules

$$
0 \rightarrow \Lambda^{i} V_{\mathbb{C}} \rightarrow \Omega_{p}^{i, 0} \stackrel{d_{Q}}{\rightarrow} \Omega_{p}^{i, 1} \stackrel{d_{Q}}{\rightarrow} \ldots \stackrel{d_{Q}}{\rightarrow} \Omega_{p}^{i, n-i-1} \stackrel{\nu}{\rightarrow} \mathbf{V a l}_{i}^{\infty} \rightarrow 0 .
$$

\section{Proof of Theorems 1 and $\mathbf{1}^{\prime}$}

Theorems 1 and $1^{\prime}$ are just reformulations of each other. We give the proof of Theorem 1 first and then show how Theorem $1^{\prime}$ follows from it.

Proof of Theorem [1: The cases $i=0$ and $i=n$ are trivial. Moreover, by Theorem 2.4, we may assume that $n / 2 \leq i<n$.

Let $\Gamma_{\lambda}$ be an arbitrary irreducible $\mathrm{SO}(n)$ module of highest weight $\lambda=\left(\lambda_{1}, \ldots, \lambda_{\lfloor n / 2\rfloor}\right)$. It is well known (and a consequence of Corollary 4.2) that the multiplicity of $\Gamma_{\lambda}$ in the spaces $\Omega_{p}^{i, j}$ of primitive forms is finite. The same holds true for the spaces $\mathbf{V a l}_{i}$ since they are quotients of $\Omega_{p}^{i, n-i-1}$ by Theorem 4.3. Thus, by Theorem 4.3, we have

$$
m\left(\mathbf{V a l}_{i}, \lambda\right)=(-1)^{n-i} m\left(\Lambda^{i} V_{\mathbb{C}}, \lambda\right)+\sum_{j=0}^{n-i-1}(-1)^{n-1-i-j} m\left(\Omega_{p}^{i, j}, \lambda\right),
$$

where $m(\cdot, \lambda)$ denotes the multiplicity of $\Gamma_{\lambda}$ in the respective $\mathrm{SO}(n)$ modules.

Let $W \cong W^{*}$ denote the (complex) standard representation of $\mathrm{SO}(n-1)$. By Corollary 4.2 and (3.4), we have

$m\left(\Omega_{p}^{i, j}, \lambda\right)=m\left(\operatorname{Ind}_{\mathrm{SO}(n-1)}^{\mathrm{SO}(n)}\left(\Lambda^{i} W \otimes \Lambda^{j} W\right), \lambda\right)-m\left(\operatorname{Ind}_{\mathrm{SO}(n-1)}^{\mathrm{SO}(n)}\left(\Lambda^{i-1} W \otimes \Lambda^{j-1} W\right), \lambda\right)$.

Thus, it follows from an application of Corollary 3.4 (with $n$ replaced by $n-1$ and $0 \leq j \leq n-1-i$ ) that

$$
m\left(\Omega_{p}^{i, j}, \lambda\right)=\sum_{\sigma} m\left(\operatorname{Ind}_{\mathrm{SO}(n-1)}^{\mathrm{SO}(n)} \bar{\Gamma}_{\sigma}, \lambda\right),
$$

where the sum ranges over all $k:=\lfloor(n-1) / 2\rfloor$-tuples of non-negative highest weights $\sigma=\left(\sigma_{1}, \ldots, \sigma_{k}\right)$ of $\mathrm{SO}(n-1)$ such that

$$
\sigma_{1} \leq 2, \quad \#(\sigma, 1)=n-1-i-j, \quad \#(\sigma, 2) \leq j .
$$


If $\mathcal{P}_{i}$ denotes the union of these $k$-tuples of non-negative highest weights of $\mathrm{SO}(n-1)$, then, by (5.1) and (5.2),

$$
m\left(\mathbf{V a l}_{i}, \lambda\right)=(-1)^{n-i} m\left(\Lambda^{i} V_{\mathbb{C}}, \lambda\right)+\sum_{\sigma \in \mathcal{P}_{i}}(-1)^{|\sigma|} m\left(\operatorname{Ind}_{\mathrm{SO}(n-1)}^{\mathrm{SO}(n)} \bar{\Gamma}_{\sigma}, \lambda\right)
$$

Let $\lambda^{*}=\left(\lambda_{1}^{*}, \ldots, \lambda_{\lfloor n / 2\rfloor}^{*}\right)$, where $\lambda_{1}^{*}:=\min \left\{\lambda_{1}, 2\right\}$ and $\lambda_{j}^{*}:=\left|\lambda_{j}\right|$ for every $1<j \leq\lfloor n / 2\rfloor$. By Theorem 3.5, Theorem 3.6 and the definition of $\bar{\Gamma}_{\sigma}$, we have

$$
\sum_{\sigma \in \mathcal{P}_{i}}(-1)^{|\sigma|} m\left(\operatorname{Ind}_{\mathrm{SO}(n-1)}^{\mathrm{SO}(n)} \bar{\Gamma}_{\sigma}, \lambda\right)=\sum_{\mu}(-1)^{|\mu|},
$$

where the sum on the right ranges over all sequences $\mu=\left(\mu_{1}, \ldots, \mu_{k}\right)$ with $\mu_{n-i}=0$ and

$$
\begin{cases}\lambda_{1}^{*} \geq \mu_{1} \geq \lambda_{2}^{*} \geq \mu_{2} \geq \ldots \geq \mu_{k-1} \geq \lambda_{\lfloor n / 2\rfloor}^{*} \geq\left|\mu_{k}\right| & \text { for odd } n, \\ \lambda_{1}^{*} \geq \mu_{1} \geq \lambda_{2}^{*} \geq \mu_{2} \geq \ldots \geq \mu_{k} \geq \lambda_{n / 2}^{*} & \text { for even } n .\end{cases}
$$

If $\lambda_{n-i+1}^{*}>0$, there is no such sequence. If $\lambda_{n-i+1}^{*}=0$, we obtain

$$
\sum_{\sigma \in \mathcal{P}_{i}}(-1)^{|\sigma|} m\left(\operatorname{Ind}_{\mathrm{SO}(n-1)}^{\mathrm{SO}(n)} \bar{\Gamma}_{\sigma}, \lambda\right)=\prod_{j=1}^{n-i-1} \sum_{\mu_{j}=\lambda_{j+1}^{*}}^{\lambda_{j}^{*}}(-1)^{\mu_{j}}
$$

This product vanishes if the $\lambda_{j}^{*}, j=1, \ldots, n-i$, do not all have the same parity. If the $\lambda_{j}^{*}, j=1, \ldots, n-i$, all have the same parity, the product above equals $(-1)^{(n-i-1) \lambda_{1}^{*}}$. Consequently, we obtain for $i>n / 2$,

$$
\sum_{\sigma \in \mathcal{P}_{i}}(-1)^{|\sigma|} m\left(\operatorname{Ind}_{\mathrm{SO}(n-1)}^{\mathrm{SO}(n)} \bar{\Gamma}_{\sigma}, \lambda\right)= \begin{cases}(-1)^{n-i-1} & \text { if } \Gamma_{\lambda} \cong \Lambda^{n-i} V_{\mathbb{C}} \\ 1 & \text { if } \lambda \text { satisfies (i), (ii), (iii) } \\ 0 & \text { otherwise }\end{cases}
$$

If $i=n / 2$, in which case $n$ is even, then

$$
\sum_{\sigma \in \mathcal{P}_{i}}(-1)^{|\sigma|} m\left(\operatorname{Ind}_{\mathrm{SO}(n-1)}^{\mathrm{SO}(n)} \bar{\Gamma}_{\sigma}, \lambda\right)= \begin{cases}(-1)^{i-1} & \text { if } \lambda=(1, \ldots, 1, \pm 1) \\ 1 & \text { if } \lambda \text { satisfies (i), (ii) and (iii) } \\ 0 & \text { otherwise. }\end{cases}
$$

Plugging this into (5.3) and using that $\Lambda^{n / 2} V_{\mathbb{C}}=\Gamma_{(1, \ldots, 1)} \oplus \Gamma_{(1, \ldots, 1,-1)}$ if $n$ is even and $\Lambda^{n-i} V_{\mathbb{C}} \cong \Lambda^{i} V_{\mathbb{C}}$ for every $i \in\{0, \ldots, n\}$, completes the proof. 
Next we explain how Theorem 1' can be deduced from Theorem 1. The argument presented here in fact shows that Theorem 1 and $1^{\prime}$ are equivalent.

Proof of Theorem $1^{\prime}$ : Let $\Gamma=\Gamma_{\mu}$ be an irreducible $\mathrm{SO}(n)$-module. The space of $\Gamma$-valued valuations is isomorphic to $\mathbf{V a l} \otimes \Gamma$.

Let $S$ denote the set of highest weights of $\mathrm{SO}(n)$ satisfying conditions (i)-(iii). By Theorem 1, we have

$$
\operatorname{dim}\left(\mathbf{V a l}_{i} \otimes \Gamma\right)^{\mathrm{SO}(n)}=\operatorname{dim}\left(\mathbf{V a l}_{i}^{f} \otimes \Gamma\right)^{\mathrm{SO}(n)}=\sum_{\lambda \in S} \operatorname{dim}\left(\Gamma_{\lambda} \otimes \Gamma_{\mu}\right)^{\mathrm{SO}(n)} .
$$

Here and in the following, the superscript $\mathrm{SO}(n)$ denotes the subspaces of $\mathrm{SO}(n)$ invariant elements. The $\Gamma_{\lambda}, \lambda \in S$ are not necessarily self dual (compare Lemma 3.2). However, if $\lambda \in S$, then also $\lambda^{\prime} \in S$, where $\lambda^{\prime}$ is the highest weight of $\Gamma_{\lambda}^{*}$. Thus, by Schur's lemma, we have

$$
\operatorname{dim}\left(\mathbf{V a l}_{i} \otimes \Gamma\right)^{\mathrm{SO}(n)}=\sum_{\lambda \in S} \operatorname{dim} \operatorname{Hom}_{\mathrm{SO}(n)}\left(\Gamma_{\lambda}, \Gamma_{\mu}\right)= \begin{cases}1 & \text { if } \mu \in S ; \\ 0 & \text { otherwise }\end{cases}
$$

\section{Examples:}

(a) If $\Gamma=\Gamma_{(0, \ldots, 0)} \cong \mathbb{C}$ is the trivial representation, then $(\mathbf{V a l} \otimes \Gamma)^{\mathrm{SO}(n)} \cong$ $\mathrm{Val}^{\mathrm{SO}(n)}$ is the vector space of all continuous rigid motion invariant valuations and Theorem 1' reduces to Hadwiger's characterization of intrinsic volumes.

(b) If $\Gamma=\Gamma_{(1,0, \ldots, 0)} \cong V_{\mathbb{C}}$ is the standard representation, then there is no translation invariant and $\mathrm{SO}(n)$ equivariant continuous valuation with values in $\Gamma$.

(c) By (3.3), we have, for $1 \leq i \leq n-1$,

$$
\operatorname{dim}\left(\mathbf{V a l}_{i} \otimes \operatorname{Sym}^{k} V_{\mathbb{C}}\right)^{\operatorname{SO}(n)}= \begin{cases}k / 2+1 & \text { if } k \text { is even } \\ (k-1) / 2 & \text { if } k \text { is odd. }\end{cases}
$$

In particular, if $k=2$, then there exist (up to constant multiples) two translation invariant and $\mathrm{SO}(n)$ equivariant continuous $\mathrm{Sym}^{2} V_{\mathbb{C}}$ valued valuations of a given degree $1 \leq i \leq n-1$. These valuations are explicitly known (see [28, 33, 48]). 
(d) If $\Gamma=\Gamma_{(1,1,0, \ldots, 0)}=\Lambda^{2} V_{\mathbb{C}}$ is the space of skew-symmetric tensors of rank two, then there is no translation invariant and $\mathrm{SO}(n)$ equivariant continuous valuation with values in $\Gamma$. This answers (the translation invariant case of) a question by Yang [53].

(e) The unique translation invariant and $\mathrm{SO}(n)$ equivariant continuous valuation with values in $\Gamma_{(2, \ldots, 2,0, \ldots, 0)}$ was constructed in [11].

\section{Bivaluations}

We turn now to the study of bivaluations. In particular, we will present the proof of Theorem 2 at the end of this section.

We denote the vector space of all continuous translation biinvariant complex valued bivaluations by $\mathbf{B V a l}$ and we write $\mathbf{B V a l}{ }_{i, j}$ for its subspace of all bivaluations of bidegree $(i, j)$. An immediate consequence of McMullen's decomposition (1.1) of the vector space Val is a corresponding result for the space BVal:

Corollary 6.1. The space $\mathbf{B V a l}$ admits a decomposition

$$
\mathbf{B V a l}=\bigoplus_{i, j=0}^{n} \mathrm{BVal}_{i, j}
$$

Corollary 6.1 implies an analog of Corollary 2.1 for the space of translation biinvariant bivaluations as follows.

Corollary 6.2. Let $C \in \mathcal{K}^{n}$ be a fixed convex body with non-empty interior. The space $\mathbf{B V a l}$ becomes a Banach space under the norm

$$
\|\varphi\|=\sup \{|\varphi(K, L)|: K, L \subseteq C\} .
$$

Moreover, a different choice of $C$ yields an equivalent norm.

The group $\mathrm{O}(n) \times \mathrm{O}(n)$ acts continuously on the Banach space $\mathbf{B V a l}$ by

$$
((\eta, \vartheta) \phi)(K, L)=\phi\left(\eta^{-1} K, \vartheta^{-1} L\right), \quad(\eta, \vartheta) \in \mathrm{O}(n) \times \mathrm{O}(n), \varphi \in \text { BVal. }
$$

We denote by $\mathbf{B V a l}{ }^{f}$ the subspace of bivaluations with finite dimensional $\mathrm{O}(n) \times \mathrm{O}(n)$ orbit. Since $\mathrm{O}(n) \times \mathrm{O}(n)$ is compact, $\mathbf{B V a l}^{f}$ is a dense subspace of BVal (see e.g. [17, p. 141]). 
Proposition 6.3. Let $0 \leq i, j \leq n$. The linear map $\iota: \mathbf{V a l}_{i}^{f} \otimes \mathrm{Val}_{j}^{f} \rightarrow$ $\mathbf{B V a l}_{i, j}^{f}$, induced by

$$
\iota(\phi \otimes \psi)(K, L)=\phi(K) \psi(L)
$$

is an isomorphism of $\mathrm{O}(n) \times \mathrm{O}(n)$ modules.

Proof: It is easy to see that the map $\iota$ is $\mathrm{O}(n) \times \mathrm{O}(n)$ equivariant and injective. It remains to prove that it is onto.

It is well known that every irreducible $\mathrm{O}(n) \times \mathrm{O}(n)$ module is of the form $\Gamma \otimes \Theta$, where $\Gamma, \Theta$ are irreducible $\mathrm{O}(n)$ modules (c.f. [17, p. 82]). Thus, if $\varphi \in \mathbf{B V a l}_{i, j}^{f}$ belongs to a subspace isomorphic to $\Gamma \otimes \Theta$, the valuation $\varphi(\cdot, L) \in \mathbf{V a l}_{i}$ belongs to a subspace which is isomorphic to $\Gamma$ as an $\mathrm{O}(n)$ module for every $L \in \mathcal{K}^{n}$. Since any $\mathrm{O}(n)$ representation whose restriction to $\mathrm{SO}(n)$ is multiplicity free, is itself multiplicity free, it follows from Theorem 1 that the irreducible subspace of $\mathbf{V a l}_{i}$ which is isomorphic to $\Gamma$ has multiplicity at most one.

If $\left\{\phi_{1}, \ldots, \phi_{l}\right\}$ is a basis of the isomorphic copy of $\Gamma$ in $\mathbf{V a l}_{i}$, then

$$
\varphi(\cdot, L)=\sum_{k=1}^{l} \phi_{k}(\cdot) \psi_{k}(L),
$$

where $\psi_{k}(L)$ are coefficients depending on $L$. It is not difficult to show that $\psi_{k} \in \mathbf{V a l}_{j}$ and that $\psi_{k}$ belongs to an isomorphic copy of $\Theta$ in $\mathbf{V a l}_{j}$ for every $k \in\{1, \ldots, l\}$. Thus, we have shown that $\varphi$ is the image under the map $\iota$ of the element $\sum_{k=1}^{l} \phi_{k} \otimes \psi_{k} \in \mathbf{V a l}_{i}^{f} \otimes \mathbf{V a l}_{j}^{f}$.

After these preparations, we are now in a position to proof the following refinement of Theorem 2 .

Theorem 6.4. Suppose that $\varphi \in \mathbf{B V a l}_{i, i}$, where $0 \leq i \leq n$.

(a) If $\varphi$ is $\mathrm{O}(n)$ invariant, then $\varphi(K, L)=\varphi(L, K)$ for every $K, L \in \mathcal{K}^{n}$.

(b) If $\varphi$ is $\mathrm{SO}(n)$ invariant and $(i, n) \neq(2 k+1,4 k+2), k \in \mathbb{N}$, then $\varphi(K, L)=\varphi(L, K)$ for every $K, L \in \mathcal{K}^{n}$.

(c) If $(i, n)=(2 k+1,4 k+2), k \in \mathbb{N}$, then there exist an $\mathrm{SO}(n)$ invariant $\zeta \in \mathbf{B V a l}_{i, i}$ and $K, L \in \mathcal{K}^{n}$ such that $\zeta(K, L) \neq \zeta(L, K)$. 
Proof: Since the cases $i=0$ and $i=n$ are trivial, we may assume that $0<i<n$. Moreover, since $\mathrm{O}(n) \times \mathrm{O}(n)$ finite bivaluations are dense in $\mathbf{B V a l}_{i, i}$ we may assume that $\varphi \in \mathbf{B V a l}_{i, i}^{f}$, where $1 \leq i \leq n$.

From Theorem 1 we deduce that the decomposition of the space $\mathbf{V a l}_{i}$ into irreducible $\mathrm{O}(n)$ modules is multiplicity free, say

$$
\mathbf{V a l}_{i}=\bigoplus_{\gamma \in R} \Gamma_{\gamma}
$$

where the sum ranges over some set $R$ of equivalence classes of irreducible representations of $\mathrm{O}(n)$.

From Proposition 6.3, it follows that

$$
\mathbf{B V a l}_{i, i}^{f, \mathrm{O}(n)} \cong\left(\mathbf{V a l}_{i}^{f} \otimes \mathbf{V a l}_{i}^{f}\right)^{\mathrm{O}(n)} \cong \bigoplus_{\gamma, \delta \in R}\left(\Gamma_{\gamma} \otimes \Gamma_{\delta}\right)^{\mathrm{O}(n)}
$$

Since, by Lemma 3.2, all representations of $\mathrm{O}(n)$ are self-dual, we have

$$
\left(\Gamma_{\gamma} \otimes \Gamma_{\delta}\right)^{\mathrm{O}(n)} \cong \operatorname{Hom}_{\mathrm{O}(n)}\left(\Gamma_{\gamma}, \Gamma_{\delta}\right) \cong \operatorname{Hom}_{\mathrm{O}(n)}\left(\Gamma_{\gamma} \otimes \Gamma_{\delta}, \mathbb{C}\right) .
$$

Since $\Gamma_{\gamma}$ and $\Gamma_{\delta}$ are irreducible, Schur's lemma implies that

$$
\operatorname{dim} \operatorname{Hom}_{\mathrm{O}(n)}\left(\Gamma_{\gamma}, \Gamma_{\delta}\right)= \begin{cases}1 & \text { if } \gamma=\delta \\ 0 & \text { if } \gamma \neq \delta\end{cases}
$$

Since all representations of $\mathrm{O}(n)$ are real, the space

$$
\operatorname{Hom}_{\mathrm{O}(n)}\left(\Gamma_{\gamma} \otimes \Gamma_{\gamma}, \mathbb{C}\right)=\left(\operatorname{Sym}^{2} \Gamma_{\gamma}\right)^{\mathrm{O}(n)} \oplus\left(\Lambda^{2} \Gamma_{\gamma}\right)^{\mathrm{O}(n)}
$$

of $\mathrm{O}(n)$ invariant bilinear forms on $\Gamma_{\gamma}$ coincides with $\left(\operatorname{Sym}^{2} \Gamma_{\gamma}\right)^{\mathrm{O}(n)}$. Thus,

$$
\mathbf{B V a l}_{i, i}^{f, \mathrm{O}(n)} \cong \bigoplus_{\gamma \in R}\left(\mathrm{Sym}^{2} \Gamma_{\gamma}\right)^{\mathrm{O}(n)}
$$

which completes the proof of statement (a). 
If $n \not \equiv 2 \bmod 4$, then the proof of statement (b) is similar, since in this case all representations of $\mathrm{SO}(n)$ are also real, by Lemma 3.2. However, in the case $n \equiv 2 \bmod 4$, more care is needed, since there are $\mathrm{SO}(n)$ modules which are not real. By Lemma 3.2, an irreducible $\mathrm{SO}(n)$ module $\Gamma_{\lambda}$ of highest weight $\lambda=\left(\lambda_{1}, \ldots, \lambda_{n / 2}\right)$ is real if and only if $\lambda_{n / 2}=0$. If $i \neq n / 2$, then, by Theorem 1, all irreducible $\mathrm{SO}(n)$ modules which enter $\mathrm{Val}_{i}$ are of this form. Consequently, any $\mathrm{SO}(n)$ invariant bivaluation $\varphi \in \mathbf{B V a l}_{i, i}$ is symmetric in this case.

Finally let $n \equiv 2 \bmod 4$ and $i=n / 2$. By Theorem 1 , the dual irreducible $\mathrm{SO}(n)$ modules $\Gamma_{(2, \ldots, 2)}$ and $\Gamma_{(2, \ldots, 2,-2)}$ both enter $\mathbf{V a l}_{i}$ with multiplicity one. If $\left\{\phi_{1}, \ldots, \phi_{l}\right\}$ is a basis of $\Gamma_{(2, \ldots, 2)} \subseteq \operatorname{Val}_{i}^{f}$ and $\left\{\psi_{1}, \ldots, \psi_{l}\right\}$ denotes the corresponding dual basis in $\Gamma_{(2, \ldots, 2,-2)} \subseteq \operatorname{Val}_{i}^{f}$, then the image of $\sum_{k=1}^{l} \phi_{k} \otimes \psi_{k}$ under the map $\iota$ defined in (6.1) clearly is a continuous $\mathrm{SO}(n)$ invariant bivaluation in $\mathbf{B V a l}_{i, i}$. However, it is not symmetric since the valuations $\left\{\phi_{1}, \ldots, \phi_{l}, \psi_{1}, \ldots, \psi_{l}\right\}$ are linearly independent.

\section{Applications to geometric inequalities}

As applications of Theorem 2, we present in this section several new geometric inequalities involving $\mathrm{SO}(n)$ equivariant Minkowski valuations. Their proofs are based, on one hand, on the symmetry of bivaluations and, on the other hand, on techniques developed by Lutwak [38-42].

Lemma 7.1. If $\Phi \in \mathrm{MVal}$ is $\mathrm{SO}(n)$ equivariant, then $\Phi$ is also $\mathrm{O}(n)$ equivariant.

Proof: Let CVal denote the vector space of all continuous and translation invariant valuations with values in the space $C\left(S^{n-1}\right)$ of continuous complex valued functions on $S^{n-1}$. Note that any $\mathrm{SO}(n)$ equivariant $\Phi \in$ MVal induces an $\mathrm{SO}(n)$ equivariant $\bar{\Phi} \in \mathbf{C V a l}$, by $\bar{\Phi}(K, \cdot)=h(\Phi K, \cdot)$. Therefore, it is sufficient to show that any $\mathrm{SO}(n)$ equivariant valuation in $\mathbf{C V a l}$ is $\mathrm{O}(n)$ equivariant.

Using arguments as in the proof of Proposition 6.3, it is easy to show that $\mathbf{C V a l}^{f} \cong \mathbf{V a l}^{f} \otimes C\left(S^{n-1}\right)^{f}$ as $\mathrm{O}(n) \times \mathrm{O}(n)$ modules. Consequently,

$$
\mathbf{C V a l}^{f, \mathrm{O}(n)} \cong\left(\mathrm{Val}^{f} \otimes C\left(S^{n-1}\right)^{f}\right)^{\mathrm{O}(n)} .
$$


It is well known that the decomposition of $C\left(S^{n-1}\right)$ into irreducible $\mathrm{SO}(n)$ modules is given by

$$
C\left(S^{n-1}\right)=\bigoplus_{k \geq 0} \Gamma_{(k, 0, \ldots, 0)},
$$

where the spaces $\Gamma_{(k, 0, \ldots, 0)}$ are precisely the spaces of spherical harmonics of degree $k$ in dimension $n$. Moreover, the spaces $\Gamma_{(k, 0, \ldots, 0)}$ are self-dual and $\mathrm{O}(n)$ invariant and, thus, (7.2) also represents the decomposition of $C\left(S^{n-1}\right)$ into irreducible $\mathrm{O}(n)$ modules.

Let $m_{k}$ denote the (finite) multiplicity of the isomorphic copy of $\Gamma_{(k, 0, \ldots, 0)}$ in Val. From (7.1), Theorem 1 and an application of Schur's lemma, we obtain

$$
\mathbf{C V a l}^{\mathrm{SO}(n)}=\bigoplus_{k} m_{k}\left(\Gamma_{(k, 0, \ldots, 0)} \otimes \Gamma_{(k, 0, \ldots, 0)}\right)^{\mathrm{SO}(n)}=\mathbf{C V a l}^{\mathrm{O}(n)} .
$$

Thus, any $\mathrm{SO}(n)$ equivariant valuation in $\mathbf{C V a l}$ is also $\mathrm{O}(n)$ equivariant.

For $K, L \in \mathcal{K}^{n}$ and $0 \leq i \leq n-1$, we write $W_{i}(K, L)$ to denote the mixed volume $V(K, \ldots, K, B, \ldots, B, L)$, where $K$ appears $n-1-i$ times and the Euclidean unit ball $B$ appears $i$ times. The mixed volume $W_{i}(K, K)$ will be written as $W_{i}(K)$ and is called the $i$-th quermassintegral of $K$. The $i$-th intrinsic volume $V_{i}(K)$ of $K$ is defined by

$$
\kappa_{n-i} V_{i}(K)=\left(\begin{array}{c}
n \\
i
\end{array}\right) W_{n-i}(K),
$$

where $\kappa_{n}$ is the $n$-dimensional volume of the Euclidean unit ball in $V$.

We will repeatedly make use of the following consequence of Theorem 2 and Lemma 7.1 .

Corollary 7.2. If $\Phi_{i} \in \mathbf{M V a l}_{i}, 1 \leq i \leq n-1$, is $\mathrm{SO}(n)$ equivariant, then

$$
W_{n-1-i}\left(K, \Phi_{i} L\right)=W_{n-1-i}\left(L, \Phi_{i} K\right)
$$

for every $K, L \in \mathcal{K}^{n}$.

Let $\mathcal{K}_{\mathrm{o}}^{n} \subseteq \mathcal{K}^{n}$ denote the set of convex bodies with non-empty interior. One of the fundamental inequalities for mixed volumes is the (general) Minkowski inequality: If $K, L \in \mathcal{K}_{\mathrm{o}}^{n}$ and $0 \leq i \leq n-2$, then

$$
W_{i}(K, L)^{n-i} \geq W_{i}(K)^{n-i-1} W_{i}(L),
$$

with equality if and only if $K$ and $L$ are homothetic. 
Lemma 7.3. Let $\Phi_{i} \in \mathbf{M V a l}_{i}, 1 \leq i \leq n-1$, be $\mathrm{SO}(n)$ equivariant and non-trivial, i.e. $\Phi_{i}(K) \neq\{0\}$ for some $K \in \mathcal{K}^{n}$.

(a) There exists a constant $r\left(\Phi_{i}\right)>0$ such that for every $K \in \mathcal{K}^{n}$,

$$
W_{n-1}\left(\Phi_{i} K\right)=r\left(\Phi_{i}\right) W_{n-i}(K) .
$$

(b) If $K \in \mathcal{K}_{\mathrm{o}}^{n}$, then

$$
W_{n-i}(K)^{i+1} \geq \frac{\kappa_{n}^{i}}{r\left(\Phi_{i}\right)^{i+1}} W_{n-1-i}\left(\Phi_{i} K\right) .
$$

If $\Phi_{i} \mathcal{K}_{\mathrm{o}}^{n} \subseteq \mathcal{K}_{\mathrm{o}}^{n}$, then equality holds if and only if $\Phi_{i} K$ is a ball.

Proof: Statement (a) follows from Hadwiger's characterization theorem. From repeated application of Minkowski's inequality (7.4) with $L=B$, we obtain the inequality

$$
W_{n-1}(K)^{i+1} \geq \kappa_{n}^{i} W_{n-1-i}(K),
$$

where, for $K \in \mathcal{K}_{\mathrm{o}}^{n}$, there is equality if and only if $K$ is a ball. Taking $\Phi_{i} K$ instead of $K$ and using (a), yields statement (b).

Special cases of Lemma 7.3 were previously obtained by Lutwak [38] (for $\left.\Phi_{i}=\Pi_{i}\right)$ and one of the authors [50] (for $i=n-1$ ).

In order to proof Theorem 3, we need a further generalization of the Brunn-Minkowski inequality (1.3) (where the equality conditions are not yet known): If $0 \leq i \leq n-2, K, L, K_{1}, \ldots, K_{i} \in \mathcal{K}^{n}$ and $\mathbf{C}=\left(K_{1}, \ldots, K_{i}\right)$, then

$$
V_{i}(K+L, \mathbf{C})^{1 /(n-i)} \geq V_{i}(K, \mathbf{C})^{1 /(n-i)}+V_{i}(L, \mathbf{C})^{1 /(n-i)} .
$$

Proof of Theorem [3: Since translation invariant continuous Minkowski valuations which are homogeneous of degree one are linear with respect to Minkowski addition (see e.g. [27]), the case $i=1$ is a direct consequence of inequality (1.3). Thus, we may assume that $i \geq 2$.

By Corollary 7.2 and (7.5), we have for $Q \in \mathcal{K}_{\mathrm{o}}^{n}$,

$$
\begin{aligned}
W_{n-1-i}\left(Q, \Phi_{i}(K+L)\right)^{1 / i} & =W_{n-1-i}\left(K+L, \Phi_{i} Q\right)^{1 / i} \\
& \geq W_{n-1-i}\left(K, \Phi_{i} Q\right)^{1 / i}+W_{n-1-i}\left(L, \Phi_{i} Q\right)^{1 / i} \\
& =W_{n-1-i}\left(Q, \Phi_{i} K\right)^{1 / i}+W_{n-1-i}\left(Q, \Phi_{i} L\right)^{1 / i} .
\end{aligned}
$$


It follows from Minkowski's inequality (7.4), that

$$
W_{n-1-i}\left(Q, \Phi_{i} K\right)^{i+1} \geq W_{n-1-i}(Q)^{i} W_{n-1-i}\left(\Phi_{i} K\right)
$$

and

$$
W_{n-1-i}\left(Q, \Phi_{i} L\right)^{i+1} \geq W_{n-1-i}(Q)^{i} W_{n-1-i}\left(\Phi_{i} L\right) .
$$

Thus, if we set $Q=\Phi_{i}(K+L)$ and use (7.3) , we obtain the desired inequality

$$
W_{n-1-i}\left(\Phi_{i}(K+L)\right)^{1 / i(i+1)} \geq W_{n-1-i}\left(\Phi_{i} K\right)^{1 / i(i+1)}+W_{n-1-i}\left(\Phi_{i} L\right)^{1 / i(i+1)} .
$$

Suppose now that equality holds and that $\Phi_{i} \mathcal{K}_{\mathrm{o}}^{n} \subseteq \mathcal{K}_{\mathrm{o}}^{n}$. By Theorem $1^{\prime}$, applied to the standard representation $V=\Gamma_{(1,0, \ldots, 0)}$, the Steiner point of $\Phi_{i} K$ is the origin for every $K \in \mathcal{K}^{n}$. Thus, we can deduce from the equality conditions of (7.6) and (17.7), that there exist $\lambda_{1}, \lambda_{2}>0$ such that

$$
\Phi_{i} K=\lambda_{1} \Phi_{i}(K+L) \quad \text { and } \quad \Phi_{i} L=\lambda_{2} \Phi_{i}(K+L)
$$

and

$$
\lambda_{1}^{1 / i}+\lambda_{2}^{1 / i}=1
$$

Using Lemma 7.3 (a) and (7.8) we get

$$
W_{n-i}(K+L)^{1 / i}=W_{n-i}(K)^{1 / i}+W_{n-i}(L)^{1 / i}
$$

which, by (1.3), implies that $K$ and $L$ are homothetic.

The major open problem concerning the rigid motion invariant quantities $W_{n-1-i}\left(\Phi_{i} K\right)$ is how to estimate them from below in terms of $W_{n-1-i}(K)$. A standard method of proof for isoperimetric problems of this kind was introduced by Lutwak [39] and is now known as the class reduction technique. Our last result shows how Corollary 7.2 allows for applications of the class reduction technique to the functionals $W_{n-1-i}\left(\Phi_{i} K\right), K \in \mathcal{K}^{n}$.

In the following we use $\Phi_{i}^{2} K$ to denote $\Phi_{i} \Phi_{i} K$.

Theorem 7.4. Let $\Phi_{i} \in \mathbf{M V a l}_{i}, 1 \leq i \leq n-1$, be $\mathrm{SO}(n)$ equivariant and suppose that $\Phi_{i} \mathcal{K}_{\mathrm{o}}^{n} \subseteq \mathcal{K}_{\mathrm{o}}^{n}$. If $K \in \mathcal{K}_{\mathrm{o}}^{n}$, then

$$
\frac{W_{n-1-i}\left(\Phi_{i} K\right)}{W_{n-1-i}(K)^{i}} \geq \frac{W_{n-1-i}\left(\Phi_{i}^{2} K\right)}{W_{n-1-i}\left(\Phi_{i} K\right)^{i}},
$$

with equality if and only if $K$ and $\Phi_{i}^{2} K$ are homothetic. 
Proof: Let $K, Q \in \mathcal{K}_{\mathrm{o}}^{n}$. From Corollary 7.2 and the Minkowski inequality (7.4), we obtain

$$
W_{n-1-i}\left(Q, \Phi_{i} K\right)^{i+1}=W_{n-1-i}\left(K, \Phi_{i} Q\right)^{i+1} \geq W_{n-1-i}(K)^{i} W_{n-1-i}\left(\Phi_{i} Q\right),
$$

with equality if and only if $K$ and $\Phi_{i} Q$ are homothetic. Taking $Q=\Phi_{i} K$, yields

$$
W_{n-1-i}\left(\Phi_{i} K\right)^{i+1} \geq W_{n-1-i}(K)^{i} W_{n-1-i}\left(\Phi_{i}^{2} K\right),
$$

with equality if and only if $K$ and $\Phi_{i}^{2} K$ are homothetic.

\section{References}

[1] J. Abardia, A. Bernig, Projection bodies in complex vector spaces, Adv. Math., in press.

[2] J. F. Adams, Lectures on Lie groups, W. A. Benjamin, Inc., New York-Amsterdam, 1969.

[3] S. Alesker, Continuous rotation invariant valuations on convex sets, Ann. of Math. (2) 149 (1999), 977-1005.

[4] S. Alesker, Description of continuous isometry covariant valuations on convex sets, Geom. Dedicata 74 (1999), 241-248.

[5] S. Alesker, Description of translation invariant valuations on convex sets with solution of P. McMullen's conjecture, Geom. Funct. Anal. 11 (2001), 244-272.

[6] S. Alesker, Hard Lefschetz theorem for valuations, complex integral geometry, and unitarily invariant valuations, J. Differential Geom. 63 (2003), 63-95.

[7] S. Alesker, The multiplicative structure on polynomial valuations, Geom. Funct. Anal. 14 (2004), 1-26.

[8] S. Alesker, Theory of valuations on manifolds. I. Linear spaces, Israel J. Math. 156 (2006), 311-339.

[9] S. Alesker, A Fourier type transform on translation invariant valuations on convex sets, Israel J. Math., in press.

[10] S. Alesker and J. Bernstein, Range characterization of the cosine transform on higher Grassmannians, Adv. Math. 184 (2004), 367-379.

[11] A. Bernig, Curvature tensors of singular spaces, Diff. Geom. Appl. 24 (2006), 191208.

[12] A. Bernig, A Hadwiger-type theorem for the special unitary group, Geom. Funct. Anal. 19 (2009), 356-372.

[13] A. Bernig, Invariant valuations on quaternionic vector spaces, J. Inst. de Math. de Jussieu, in press. 
[14] A. Bernig and L. Bröcker, Valuations on manifolds and Rumin cohomology, J. Differential Geom. 75 (2007), 433-457.

[15] A. Bernig and J.H.G. Fu, Convolution of convex valuations, Geom. Dedicata 123 (2006), 153-169.

[16] A. Bernig and J.H.G. Fu, Hermitian integral geometry, Ann. of Math. (2), in press.

[17] T. Bröcker, T. tom Dieck, Representations of compact Lie groups. Graduate Texts in Mathematics 98, Springer-Verlag, New York, 1985.

[18] J.H.G. Fu, Structure of the unitary valuation algebra, J. Differential Geom. 72 (2006), 509-533.

[19] W. Fulton and J. Harris, Representation theory. A first course, Graduate Texts in Mathematics 129, Springer-Verlag, New York, 1991.

[20] P. Goodey and G. Zhang, Inequalities between projection functions of convex bodies, Amer. J. Math. 120 (1998), 345-367.

[21] R. Goodman and N.R. Wallach, Representations and invariants of the classical groups, Encyclopedia of Mathematics and its Applications 68, Cambridge University Press, Cambridge, 1998.

[22] E. Grinberg and G. Zhang, Convolutions, transforms, and convex bodies, Proc. London Math. Soc. (3) 78 (1999) 77-115.

[23] C. Haberl, $L_{p}$ intersection bodies, Adv. Math. 217 (2008), 2599-2624.

[24] C. Haberl, Blaschke valuations, Amer. J. Math., in press.

[25] C. Haberl and F.E. Schuster, General $L_{p}$ affine isoperimetric inequalities, J. Differential Geom. 83 (2009), 1-26.

[26] H. Hadwiger, Translationsinvariante, additive und stetige Eibereichfunktionale, Publ. Math. Debrecen 2 (1951), 81-94.

[27] H. Hadwiger, Vorlesungen über Inhalt, Oberfläche und Isoperimetrie, Springer, Berlin, 1957.

[28] D. Hug, R. Schneider and R. Schuster, The space of isometry covariant tensor valuations, Algebra i Analiz 19 (2007), 194-224.

[29] D. Hug, R. Schneider and R. Schuster, Integral geometry of tensor valuations, Adv. in Appl. Math. 41 (2008), 482-509.

[30] M. Kiderlen, Blaschke- and Minkowski-Endomorphisms of convex bodies, Trans. Amer. Math. Soc. 358 (2006), 5539-5564.

[31] D.A. Klain and G.-C. Rota, Introduction to geometric probability, Cambridge University Press, Cambridge, 1997.

[32] M. Ludwig, Projection bodies and valuations, Adv. Math. 172 (2002), 158-168.

[33] M. Ludwig, Ellipsoids and matrix valued valuations, Duke Math. J. 119 (2003), 159-188.

[34] M. Ludwig, Minkowski valuations, Trans. Amer. Math. Soc. 357 (2005) 4191-4213.

[35] M. Ludwig, Intersection bodies and valuations, Amer. J. Math. 128 (2006), 14091428.

[36] M. Ludwig, Minkowski areas and valuations, J. Differential Geom., in press. 
[37] M. Ludwig and M. Reitzner, A classification of SL( $n)$ invariant valuations, Ann. of Math. (2) 172 (2010), 1223-1271.

[38] E. Lutwak, Mixed projection inequalities, Trans. Amer. Math. Soc. 287 (1985), 91105.

[39] E. Lutwak, On some affine isoperimetric inequalities, J. Differential Geom. 23 (1986), 1-13.

[40] E. Lutwak, Intersection bodies and dual mixed volumes, Adv. Math. 71 (1988), 232 261.

[41] E. Lutwak, Centroid bodies and dual mixed volumes, Proc. London Math. Soc. 60 (1990), 365-391.

[42] E. Lutwak, Inequalities for mixed projection bodies, Trans. Amer. Math. Soc. 339 (1993), no. 2, 901-916.

[43] E. Lutwak, D. Yang, and G. Zhang, $L_{p}$ affine isoperimetric inequalities, J. Differential Geom. 56 (2000), 111-132.

[44] E. Lutwak, D. Yang, and G. Zhang, A new ellipsoid associated with convex bodies, Duke Math. J. 104 (2000) 375-390.

[45] E. Lutwak and G. Zhang, Blaschke-Santaló inequalities, J. Differential Geom. 47 (1997), 1-16.

[46] P. McMullen, Valuations and Euler-type relations on certain classes of convex polytopes, Proc. London Math. Soc. 35 (1977), 113-135.

[47] P. McMullen, Valuations and dissections, Handbook of Convex Geometry, Vol. B (P.M. Gruber and J.M. Wills, eds.), North-Holland, Amsterdam, 1993, pp. 933-990.

[48] P. McMullen, Isometry covariant valuations on convex bodies, Rend. Circ. Mat. Palermo (2) Suppl. 50 (1997), 259-271.

[49] M. Rumin, Formes différentielles sur les variétés de contact, J. Differential Geom. 39 (1994), 281-330.

[50] F.E. Schuster, Convolutions and multiplier transformations, Trans. Amer. Math. Soc. 359 (2007), 5567-5591.

[51] F.E. Schuster, Crofton Measures and Minkowski Valuations, Duke Math. J. 154 (2010), 1-30.

[52] N.R. Wallach, Real reductive groups. I, Pure and Applied Mathematics 132, Academic Press, Inc., Boston, MA, 1988.

[53] D. Yang, Affine integral geometry from a differentiable viewpoint, Handbook of Geometric Analysis, No. 2, in press.

Tel Aviv University, IsRael

E-mail address: semyon@post.tau.ac.il

Goethe-Universität Frankfurt am Main, Germany

E-mail address: bernig@math.uni-frankfurt.de

Vienna University of Technology, Austria

E-mail address: franz.schuster@tuwien.ac.at 